\title{
Numerical and experimental characterization of the reaction rates in the core of the CNESTEN's TRIGA Mark II research reactor
}

\author{
H. Ghninou ${ }^{1}$ (hamza.ghninou @ cea.fr), A. Gruel ${ }^{1}$, A. Lyoussi ${ }^{1}$, C. Reynard-Carette ${ }^{2}$, C. El Younoussi ${ }^{3}$, \\ B. El Bakkari ${ }^{3}$, B. Nacir ${ }^{3}$, Y. Boulaich ${ }^{3}$, H. Bounouira ${ }^{3}$ \\ ${ }^{1}$ CEA, DES, IRESNE, DER (SPESI/LP2E), Cadarache F-13108 Saint-Paul-Lez-Durance, France \\ ${ }^{2}$ Aix Marseille Univ, Université de Toulon, CNRS, IM2NP, Marseille, France \\ ${ }^{3}$ CNESTEN/CENM, POB 1382, Rabat, Morocco
}

\begin{abstract}
Education, training and isotopes production are the most important uses of the Moroccan 2 MW TRIGA Mark II reactor situated at the National Center for Energy Sciences and Nuclear Techniques (CNESTEN, Morocco). To develop new $R \& D$ projects in research reactors, the particular and advanced knowledge of neutron and photon flux distribution, within and around the reactor core, is crucial. In order to precisely preparing the experiments in the CNESTEN's TRIGA reactor, a detailed model was developed using the 3D continuous energy Monte Carlo code TRIPOLI-4 and the continuous energy cross-section data from the JEFF3.1.1 nuclear data library. This new model was used to carry out preliminary neutron and photon calculations to estimate flux levels in the irradiation channels as well as to calculate kinetic parameters of the reactor, core excess reactivity, integral control rods worth and power peaking factors. As a first step of the validation of the model, the obtained results were compared with the experimental ones available in the Final Safety Analysis Report (FSAR) of the TRIGA reactor. A study is being carried out at the end of which the results will be published as an evaluated benchmark. Furthermore, this work aims at experimentally characterize the reaction rates in various irradiation channels inside and outside the reactor core. The measurements are carried out using the neutron activation technique. To set up the experimental design for the activation experiments a series of preliminary calculations were performed using the TRIPOLI-4 model to calculate the expected gamma flux/intensity levels of various materials after irradiations in different positions in the irradiation facilities. Different activation foils with known characteristics are then irradiated and the activity of several isotopes is measured with the Gamma Spectrometry Method. The measured relative reaction rates are then compared with the calculated ones evaluated through the new TRIPOLI-4 reactor model. Fairly good agreement was found, which indicates that the new computational model is accurate enough to reproduce experiments.
\end{abstract}

Keywords - TRIGA reactor, neutron activation analysis, TRIPOLI-4 simulation, $\gamma$ spectrometry.

\section{INTRODUCTION}

$\mathrm{T}$ HE precise and advanced knowledge of the neutron flux is crucial to develop new $R \& D$ projects in research reactor and to benchmark the computational models of the reactor. The Moroccan TRIGA MARK-II research reactor, operated by the CNESTEN in Rabat, is a pool type light-water reactor, with a maximum steady state power of $2 \mathrm{MW}$.

A relevant effort has been made by the CNESTEN in the past few years to accurately model the reactor to support planning, designing and implementing new experiments in various fields of nuclear research as well as to interpret and analyze the corresponding experimental results [1] [2] [3] [4].

The present paper describes the preliminary characterization of reaction rates in various irradiation and experimental channels of the CNESTEN's TRIGA Mark II research reactor. For this purpose, different metallic foils were irradiated and the resulted activities were analyzed via $\gamma$ spectrometry method. Through comparisons of measured and calculated reaction rates, the computational scheme of the reactor can be experimentally validated.

This study was conducted in the framework of the bilateral collaboration between the CEA-Cadarache and the CNESTEN.

\section{THE CNESTEN'S TRIGA MARK II REACTOR}

The 2 MW TRIGA (Training Research and Isotope production General Atomics) Mark II is a research reactor designed and manufactured by General Atomics. It is a pooltype light water moderated and cooled reactor. The reactor core is immerged into an $8 \mathrm{~m}$ high and $2.52 \mathrm{~m}$ diameter tank filled with water, it is a hexagonal array type consisting of seven concentric rings where the cylindrical fuel elements (FEs) are arranged, and two aluminum grid plates which provide accurate spacing between the FEs.

The reactor core assembly is composed of $101 \mathrm{FEs}$, including 5 fuel-follower control rods, 17 graphite elements, a central channel and a pneumatic transfer system (Fig. 1). The FEs of the TRIGA reactor are contained within cylindrical rods made of stainless steel. The height of the active fuel region of the FE is $38.1 \mathrm{~cm}$, the upper and lower endcaps are designed to enable safe positioning in the core. Two cylindrical graphite elements, serving as axial neutron reflectors, are stoppered on the top and bottom end of the fuel region (Fig. $1 \mathrm{~A}$ ). 
The fuel material consists of a uniform mixture of uranium (8.5\%wt, enriched to $19.7 \%$ of ${ }^{235} \mathrm{U}$ ) and zirconium hydride leading to a neutron moderation inside the fuel, which makes the reactor inherently safe and suitable for manpower training, nuclear research and radioisotopes production. The reactor is also equipped with a thermal column, a rotary groove and 4 beam ports channels that are clad with aluminum and filled with air. Beam port NB1 is a tangential port, whereas beam ports NB2, 3 and 4 are radially oriented ports (Fig. 2).

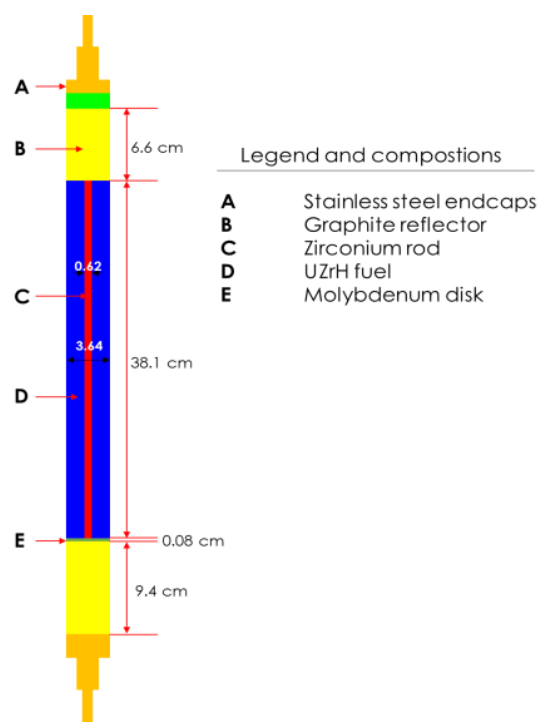

(a)

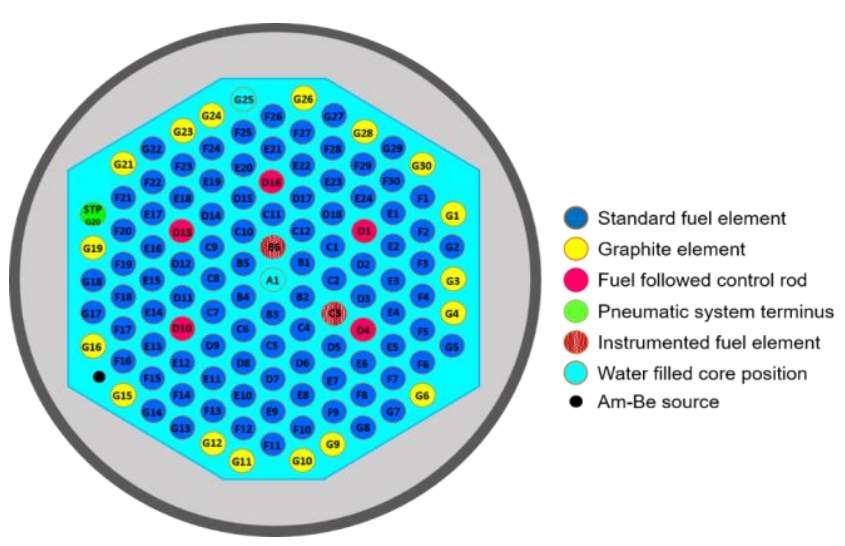

(b)

Fig. 1. (a) TRIGA standard fuel element, (b) the actual core configuration of the TRIGA reactor.

The reactor core reactivity is controlled by means of five absorbing rods, labeled SHIM I, SHIM II, SHIM III, SHIM IV and REGULATING (located in D4, D10, D13, D1 and D16 respectively). They are contained within stainless steel cylindrical rods. The lower part of the rod is filled with fuel, whereas the upper part is filled with $\mathrm{B}_{4} \mathrm{C}$ neutron absorber. When the control rods are fully inserted, the upper part with neutron absorber takes place inside the reactor at the same level of the active fueled section of fuel elements.

\section{A. Computational model}

A detailed new computational model of the TRIGA Mark II reactor was developed using the 3D continuous energy Monte
Carlo code TRIPOLI-4.11 [3] and the continuous energy cross section from the JEFF3.1.1 nuclear data library [4]. In this new model, FEs, graphite elements and control rods are modelled with full available precision, meaning that the zirconium rod, stainless steel cladding and endcaps, air gaps and molybdenum supporting disc are explicitly described. Furthermore, the supporting grids, graphite reflector, annular lead bloc, thermal column, rotary groove and beam ports are also taken into account in this model. In order to expand its utilization and to verify its validation against the MCNP reference computation model as well as against experiment [1], this new model was used to carry out preliminary neutron calculations to estimate flux levels in the irradiation channels, kinetic parameters of the reactor, control rods worth and power peaking factors. The preliminary obtained results have shown a good agreement compared to MCNP and experimental ones, and will be soon published as an evaluated criticality benchmark.

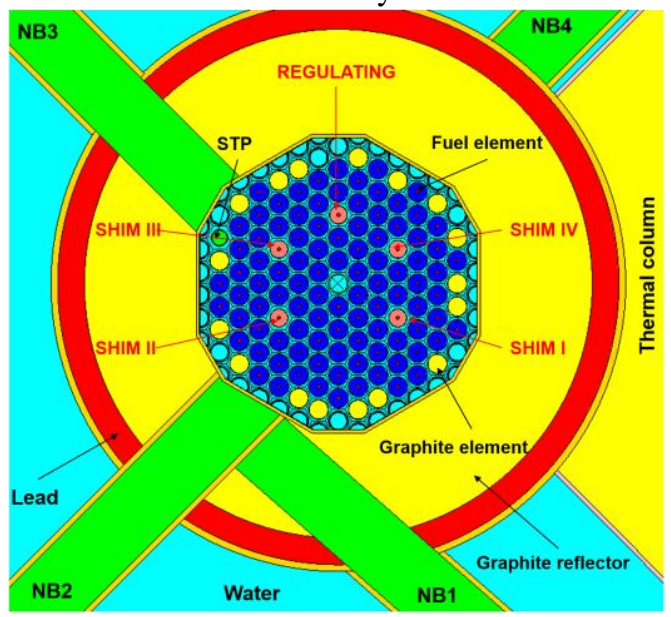

(a)

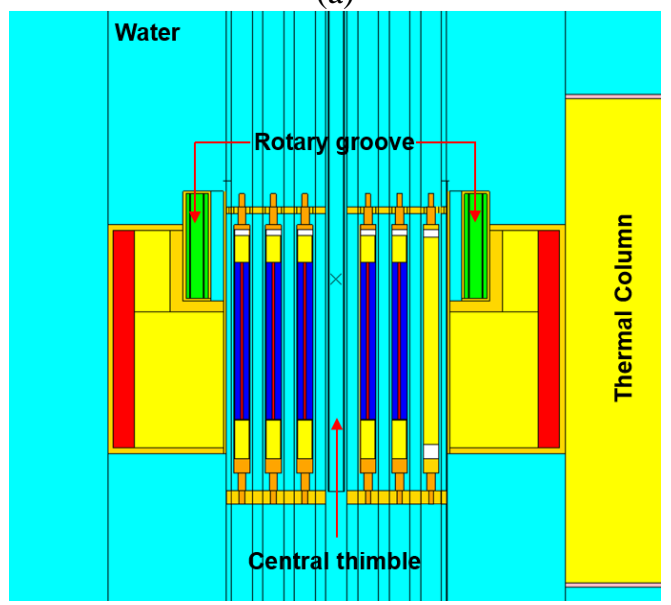

(b)

Fig. 2. (a) Side and (b) top view of the TRIGA reactor core as described in the TRIPOLI-4 model.

\section{B. Experimental design and preliminary calculations}

Preliminary simulations with the TRIPOLI-4 model were carried out in order to precisely prepare and design the experiments in the irradiation channels of the reactor. Through these simulations we can choose dosimeters that respond to a desired specific region of the neutron spectra, we can also set the optimal irradiation conditions (reactor power level and irradiations duration) in order to optimize the measurement 
time in HP-Ge detector so that we can sequence the dosimeters to be measured considering their half-lives.

Six dosimeters were selected to cover different regions in the energy range of $0-10 \mathrm{MeV}$ of the neutron spectra: ${ }^{197} \mathrm{Au}(\mathrm{n}, \gamma)$, ${ }^{59} \mathrm{Co}(\mathrm{n}, \gamma),{ }^{58} \mathrm{Ni}(\mathrm{n}, \mathrm{p}),{ }^{56} \mathrm{Fe}(\mathrm{n}, \mathrm{p}),{ }^{46} \mathrm{Ti}(\mathrm{n}, \mathrm{p})$ and ${ }^{27} \mathrm{Al}(\mathrm{n}, \alpha)$. The selection of these dosimeters is based on different criteria such as: the cross section of the neutron reaction of interest, energy range of response, energy and intensity of decay $\gamma$ rays' emission.

The reaction rates calculated in the core reactor center are shown in Fig. 3 and Fig. 4. The cumulated reaction rates are calculated by the following formula:

$$
C=\frac{\sum_{1}^{i} R_{j}}{\sum_{1}^{N} R_{j}}
$$

Where $N$ is the total number of energy groups, $R_{j}$ is a reaction rate of an energy group " $j$ ". The cumulated reaction rate for resonant dosimeters, ${ }^{197} \mathrm{Au}$ and ${ }^{59} \mathrm{Co}$, shows a large contribution of thermal neutrons. Furthermore, one might notice that the resonant dosimeters present peaks that are consistent with resonant zones of the (n, $\gamma$ ) cross section Fig. 5. Therefore, the dimensions of resonant dosimeters should be small enough so as to reduce the self-shielding and to avoid the neutron flux disturbance in the irradiation channels. Consequently, the gold foils have been chosen with a $0.1 \mathrm{~mm}$ thickness, and $1 \mathrm{~mm}$ in the case of cobalt wires.
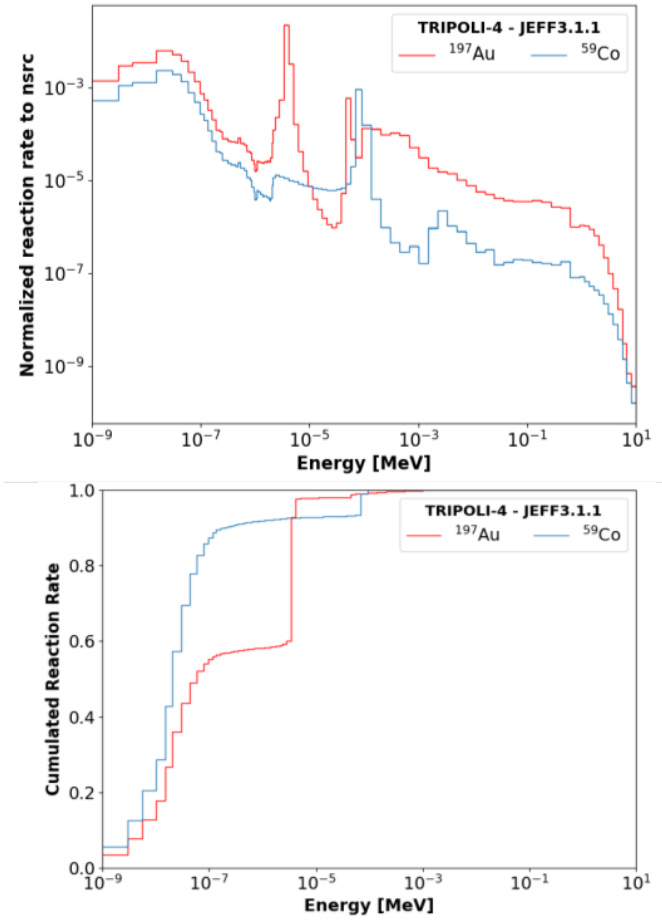

Fig. 3. Distribution of reaction rates and cumulated reaction rates for resonant dosimeters.

For threshold dosimeters, the cumulated reaction rate shows that the latter are very sensitive to the fast region of the neutron energy spectra. Threshold activation reactions, in particular (n, p) and $(n, \alpha)$ reactions, generally have a threshold energy around $1 \mathrm{MeV}$ [5]. The thickness of the threshold irradiated disk-form dosimeters was selected as follows: $0.128 \mathrm{~mm}$ for $\mathrm{Ni}$ and $\mathrm{Fe}, 0.25 \mathrm{~mm}$ for $\mathrm{Al}$ and $0.5 \mathrm{~mm}$ for $\mathrm{Ti}$.
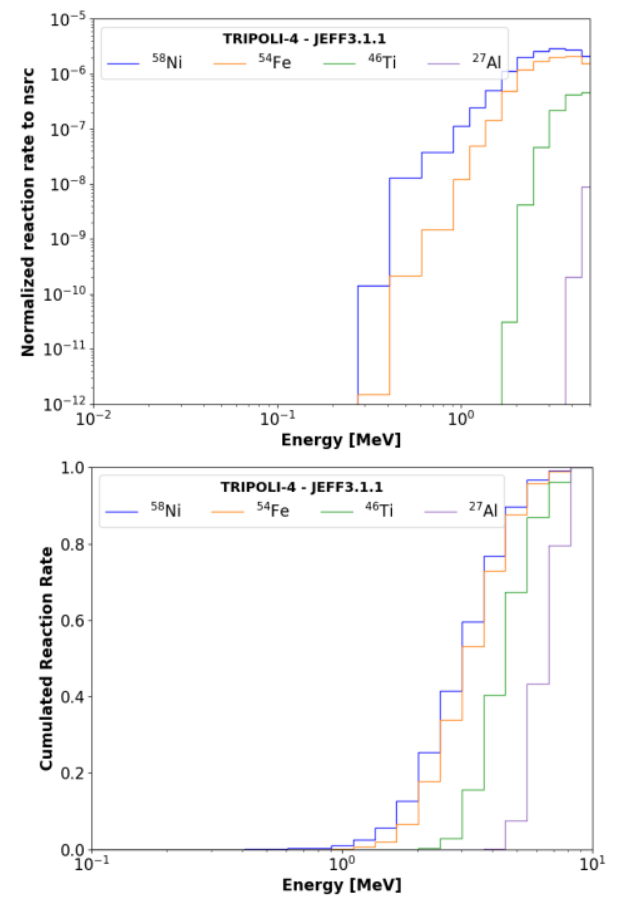

Fig. 4. Distribution of reaction rates and cumulated reaction rates for threshold dosimeters.

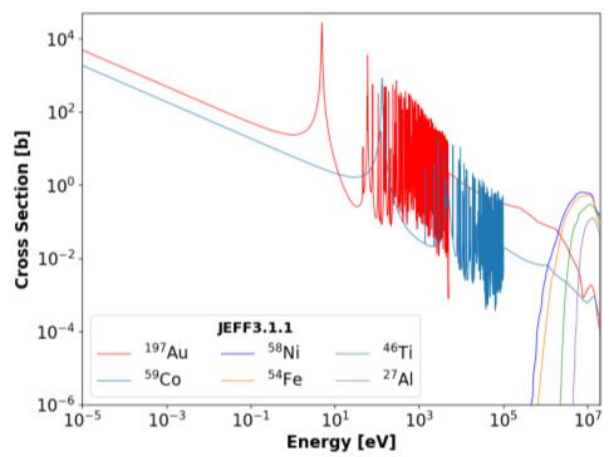

Fig. 5. Cross-sections for resonant and threshold dosimeters from JEFF3.1.1 nuclear data library [8].

\section{Experimental set-up}

The above-mentioned dosimeters were irradiated in 4 different irradiation facilities:

- Core central channel (CC) - gold and nickel dosimeters were irradiated in different axial positions in order to characterize the axial fast and thermal neutron flux profiles. For the purpose of this irradiation, a new specific device was manufactured (Fig. 6) in order to precisely position the dosimeters in this irradiation channel.

- $\quad$ Rotary groove $(\mathrm{RG})$ - gold dosimeters were irradiated in 8 symmetrical positions among the 40 circularly distributed channels, with an inner diameter of $3.8 \mathrm{~cm}$, in that irradiation facility. All six dosimeters were then irradiated in IC\#10 to characterize the neutron spectra in that position. 


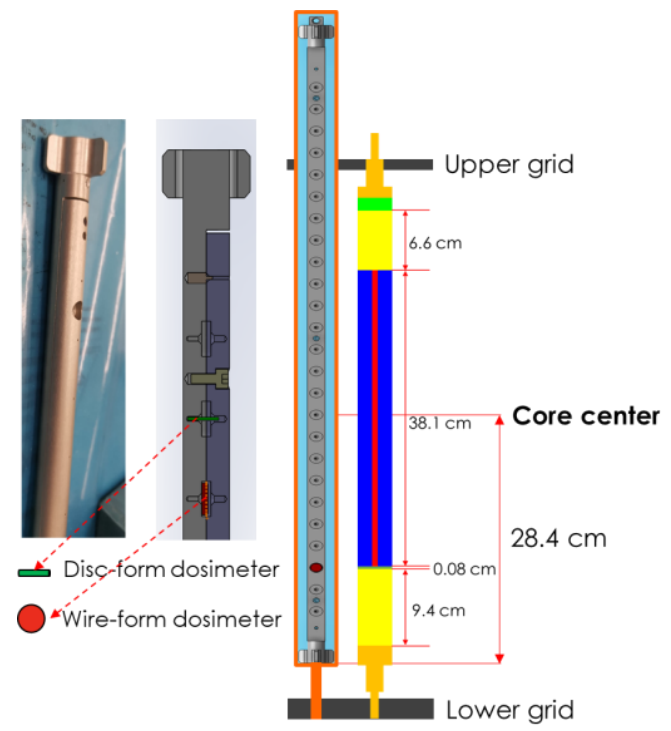

Fig. 6. experimental irradiation device in the core central channel.

- $\quad$ Pneumatic transfer system (PTS) - the PTS is used to irradiate and transport samples between the radiochemistry lab and an in-core location. As for the case of RG, all 6 dosimeters were irradiated in PTS in order to characterize spectra in that core location. The dosimeters. Before irradiation in the RG and PTS, dosimeters were prepared and fixed in different capsules depending on the irradiation facility (Fig. 7). NB1 tangential channel - gold and nickel dosimeters were irradiated in the closest position to the reactor core.
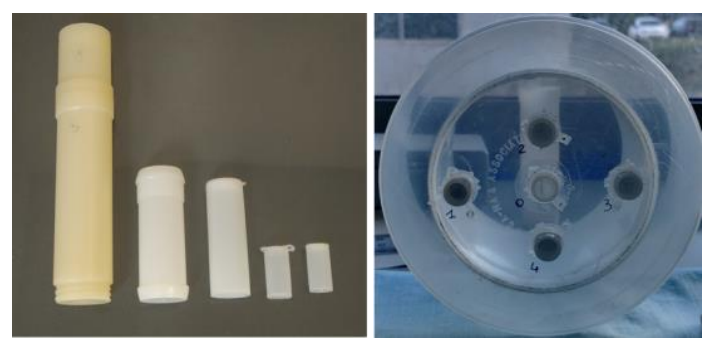

Fig. 7. (left) the specimen capsules for irradiation in PTS and RG, (right) device used for irradiation of dosimeters in the NB1 channel.

An illustration is given in Fig. 8, which shows the neutron spectra, for 315 energy groups, in the central channel, pneumatic tube and in the NB1 channel. A normalization factor was applied in order to scale the calculated flux scores to the actual reactor power level [8] :

$$
C_{M C}=\frac{P \times \bar{v}}{w_{f} \times k_{e f f}}\left[\frac{\text { neutrons }}{s}\right]
$$

With $P$ is the reactor power in [W], $\bar{v}$ is the average number of neutrons per fission, $w_{\text {eff }}$ is the energy released per fission [MeV/fission] and $k_{\text {eff }}$ is the effective multiplication factor of the reactor.

The simulations were performed in cells that have the same thicknesses as the actual dosimeters and then normalized to the full reactor power; $2 \mathrm{MW}$. The $1 \sigma$ relative statistical uncertainty, shaded area in the graph, is within $0.4 \%$ to $5 \%$ in the energy range of $10^{-10}-10 \mathrm{MeV}$.

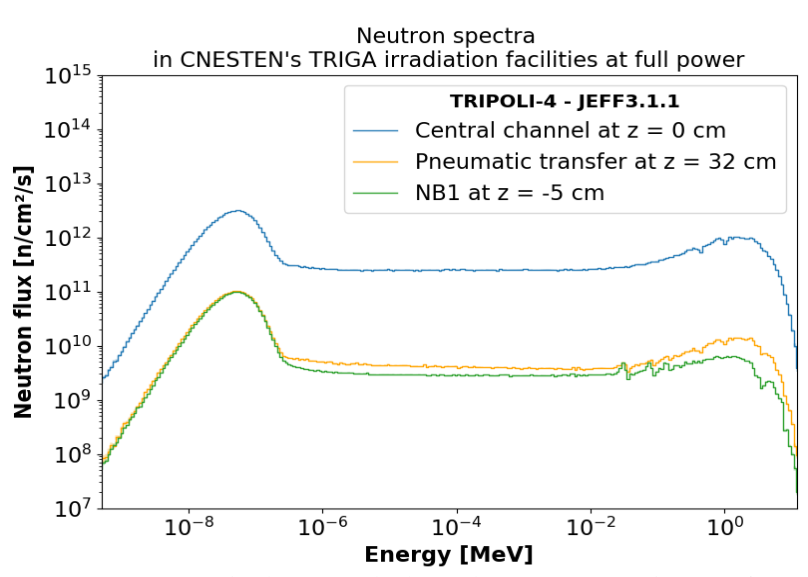

Fig. 8. Neutron spectra in the Central Channel (CC), Pneumatic Transfer System (PTS) and the tangential channel NB1.

\section{RESULTS AND DISCUSSIONS}

\section{A. Irradiations and measurements}

Four irradiations were performed, between December 2020 and March 2021, in different position within and beyond the reactor core. The irradiations lasted different times $\left(t_{i r r}\right)$ depending on the flux intensity in the irradiation facilities. The reactor power level was chosen to be $20 \mathrm{~kW}$ for irradiations of resonant dosimeters in both CC and RG (Table I). For threshold dosimeters the reactor power level was $100 \mathrm{~kW}$ and $1 \mathrm{MW}$ in the case of ${ }^{46} \mathrm{Ti}$ dosimeter irradiation.

TABLE I

THE IRRADIATIONS PERFORMED IN DIFFERENT REACTOR FACILITIES

\begin{tabular}{ccccc}
\hline $\begin{array}{c}\text { Date of } \\
\text { irradiation }\end{array}$ & Facility & Power level & $\begin{array}{c}\text { Irradiation } \\
\text { time }\end{array}$ & $\begin{array}{c}\text { Irradiated } \\
\text { samples }\end{array}$ \\
\hline $08 / 12 / 2020$ & $\begin{array}{c}\text { Center CC } \\
\text { and NB1 } \\
\text { Axial } \\
\text { profile CC } \\
\text { Axial } \\
\text { profile CC } \\
\text { and RG } \\
\text { Center CC } \\
\text { and PTS }\end{array}$ & $100 \mathrm{~kW}$ & $30 \mathrm{~min}$ & $\begin{array}{c}\mathrm{Co}, \mathrm{Fe} \text { and } \\
\mathrm{Au}\end{array}$ \\
$26 / 02 / 2021$ & $20 \mathrm{~kW}$ & $15 \mathrm{~min}$ & $\mathrm{Ni}$ and $\mathrm{Au}$ \\
$29 / 03 / 2021$ & $15 \mathrm{~min}$ & $7 \mathrm{~min} 30 \mathrm{~s}$ & $\begin{array}{c}\mathrm{Co}, \mathrm{Ni}, \mathrm{Al}, \\
\mathrm{Fe}, \mathrm{Ti}, \mathrm{Au}\end{array}$ \\
\hline \hline
\end{tabular}

The post-irradiation analysis of the irradiated dosimeters was performed by means of the wide-spread $\gamma$ spectrometry technique. In addition to the factors determined by the conditions of measurement, the activity of the radioactive products depends on neutron flux at the position of irradiation as well as the corresponding cross section of the targeted material. The relationship between the reaction rate and the activity is evinced by the following basic formula:

$$
A=N \sigma \phi\left(1-e^{-\lambda t_{i}}\right)
$$

Where $A$ is the activity produced in a dosimeter containing $N$ numbers of nuclei when irradiated for a $t_{i}$ irradiation time at a $\phi\left[\mathrm{n} \cdot \mathrm{cm}^{-2} \cdot \mathrm{s}^{-1}\right]$ neutron flux. $\sigma[\mathrm{b}]$ and $\lambda[\mathrm{s}]$ refer to the microscopic reaction cross section and the decay constant, respectively. At the end of irradiation, the activity $A_{j}$ for the $j^{\text {th }}$ peak of an isotope, in the irradiated sample, may be written as:

$$
A_{j}=\frac{N\left(E_{j}\right)}{m \times I_{\gamma}\left(E_{j}\right) \times R^{p}\left(E_{j}\right)} \times \frac{\lambda \times t}{\left(1-e^{-\lambda t}\right) \times e^{-\lambda t_{d}}} \sum_{i} C_{i}
$$

Where $N\left(E_{j}\right)$ is the net area under the total absorption peak at energy $E_{j} ; I_{\gamma}\left(E_{j}\right)$ is the emission probability of the photon with 
energy $E_{j}$ of the material in quest; $t$ is the counting time, $I_{\gamma}\left(E_{j}\right)$ data is taken from [10]; $R^{p}\left(E_{j}\right)$ is the total absorption yield for the photon with energy $E_{j ;} t_{d}$ : time between the end of irradiation and the beginning of the measurement; $m$ is the mass of the dosimeter; $\sum C_{i}$ is the sum of the different correction

factors of geometry, self-absorption of the photon of energy $E_{j}$ in the foil and summing effects due to cascade emission of photons.

The yield calibration of the CNESTEN's HP-Ge detector was carried out with a certified europium multi-gamma radioactive source of small size (considered as a punctual source) for different geometries. Depending on the dosimeter's activity, the spectrometry $\gamma$ measurements were performed at two sourcedetector distances: 21.53 and $6.93 \mathrm{~cm}$.

\section{B. Normalized values}

In this section, we present the comparison of the calculated and the measured specific activities represented as "relative quantities" e.g. normalized to a certain reference value according to the following equations:

- Profiles in central channel (CC)

$$
A_{i, \text { norm }}^{C C}=\frac{A_{i}^{C C}}{A_{\max }^{C C}}
$$

- $\quad$ Rotary groove (RG)

$$
A_{i, \text { norm }}^{R G}=\frac{A_{i}^{R G}}{\frac{1}{i_{\text {max }}} \sum_{i}^{i_{\max }} A_{i}^{R G}}
$$

- $\quad$ Pneumatic transfer system (PTS)

$$
A_{i, \text { norm }}^{\text {PTS }}=\frac{A_{i}^{\text {PTS }}}{\frac{1}{i_{\max }} \sum_{i}^{i_{\max }} A_{i}^{P T S}}
$$

Where the $A_{i}$ is the measured and calculated specific activities (in $\mathrm{Bq} / \mathrm{mg}$ ).

Figs. 9-12 refer to the comparisons of the normalized measured and calculated values. The blue area in the middle side of the graphs denotes the active fuel region of the standard FE of the TRIGA reactor and the error bars represent the $1 \sigma$ statistical and measurement uncertainties. The relative differences (C/E-1) between experimental and calculated results are presented below each graph.

The simulations were performed in criticality mode using the detailed computational TRIPOLI-4 model of TRIGA Mark II reactor (see section II.A) and JEFF3.1.1 nuclear data library. To ensure consistency between the measured and calculated values, the dosimeters and the irradiation devices were modelled explicitly inside the irradiation facilities. Furthermore, the control rod positions during the experiments were taken into account in calculations. It should be noted that the control rods travel vertically a distance of $38.1 \mathrm{~cm}$ between their fully withdrawn and inserted positions. The control rod scale is divided into 1000 steps - 0 fully inserted and 1000 fully withdrawn.

As shown in Figs. 9-10 the calculated normalized activity profiles for gold (thermal and epithermal neutron flux) and nickel (fast neutron flux) foils are in good agreement with the experimental results. For the axial distribution of the ${ }^{197} \mathrm{Au}(\mathrm{n}$, $\gamma{ }^{198} \mathrm{Au}$ reaction rate, the relative differences $(\mathrm{C} / \mathrm{E}-1)$ are around $3 \%$ in the active fuel region and around $7 \%$ in the reflector area of the FE. In the case of the ${ }^{58} \mathrm{Ni}(\mathrm{n}, \mathrm{p})^{58} \mathrm{Co}$ reaction rate, the relative differences $(\mathrm{C} / \mathrm{E}-1)$ are around 2 to $10 \%$ in the active fuel region and around $20 \%$ in the reflector parts of the FE. The preliminary analysis of the axial gold and nickel reaction rate profiles has shown a slight systematic shift in the axial direction, which was corrected by simulations. This shift may be related to a slight positioning error in the central channel.
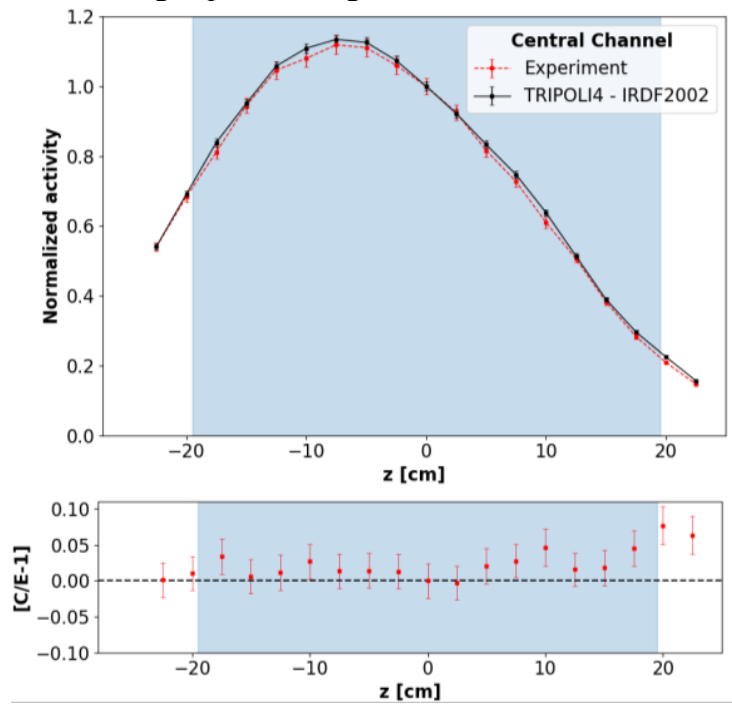

Fig. 9. Measured and calculated axial profile of ${ }^{197} \mathrm{Au}(\mathrm{n}, \gamma){ }^{198} \mathrm{Au}$ reaction rates in the reactor core central channel normalized to the maximal value. The error bars represent the $1 \sigma$ uncertainty. Area marked in blue denotes the active fuel region of the standard fuel element.
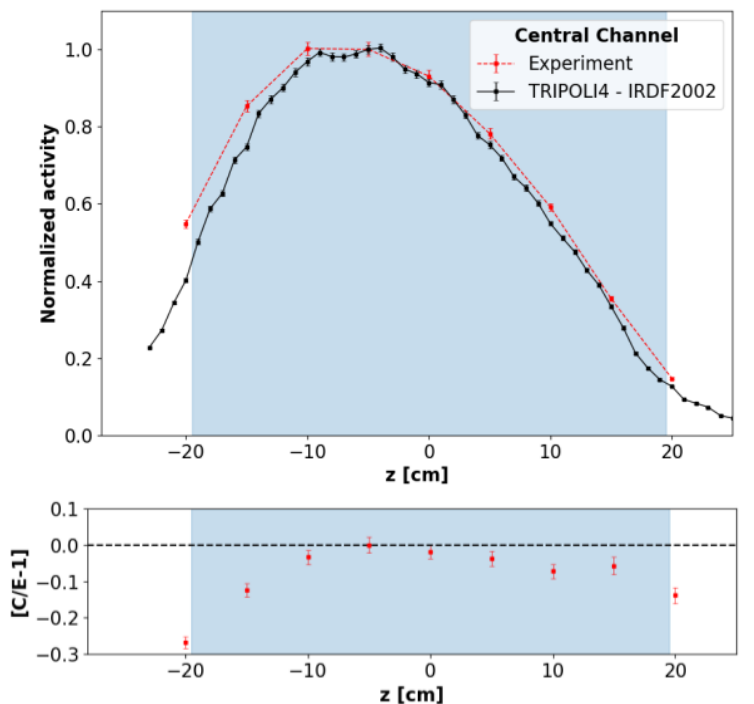

Fig. 10. Measured and calculated axial profile of ${ }^{58} \mathrm{Ni}(\mathrm{n}, \mathrm{p}){ }^{58} \mathrm{Co}$ reaction rates in the reactor core central channel normalized to the maximal value.

Fig. 11 shows a good agreement between the measured and calculated normalized gold reaction rates in 8 positions among the 40 in the rotary groove facility. The relative differences between the calculated and experimental values are in average around $6 \%(1 \sigma)$. However, the $1 \sigma$ statistical uncertainty in the calculated reaction rates have varied between $1 \%$ to $5 \%$ (in IC\#20). 
Fig. 12 displays the calculated and measured reaction rates for 5 types of dosimeters; ${ }^{197} \mathrm{Au}(\mathrm{n}, \gamma),{ }^{27} \mathrm{Al}(\mathrm{n}, \alpha),{ }^{46} \mathrm{Ti}(\mathrm{n}, \mathrm{p})$, ${ }^{58} \mathrm{Ni}(\mathrm{n}, \mathrm{p})$ and ${ }^{59} \mathrm{Co}(\mathrm{n}, \gamma)$. In order to qualitatively compare the calculated and measured values, the reaction rates were normalized to the average reaction rates of all dosimeters irradiated in the PTS (see section III.B).

A good agreement among the results was found for all dosimeters irradiated in the PTS. The C/E-1 relative differences are within $1 \%$ to $4 \%$ (maximum difference for ${ }^{46} \mathrm{Ti}$ dosimeter) which indicates that the TRIPOLI-4 model can accurately reproduce the experimental results.
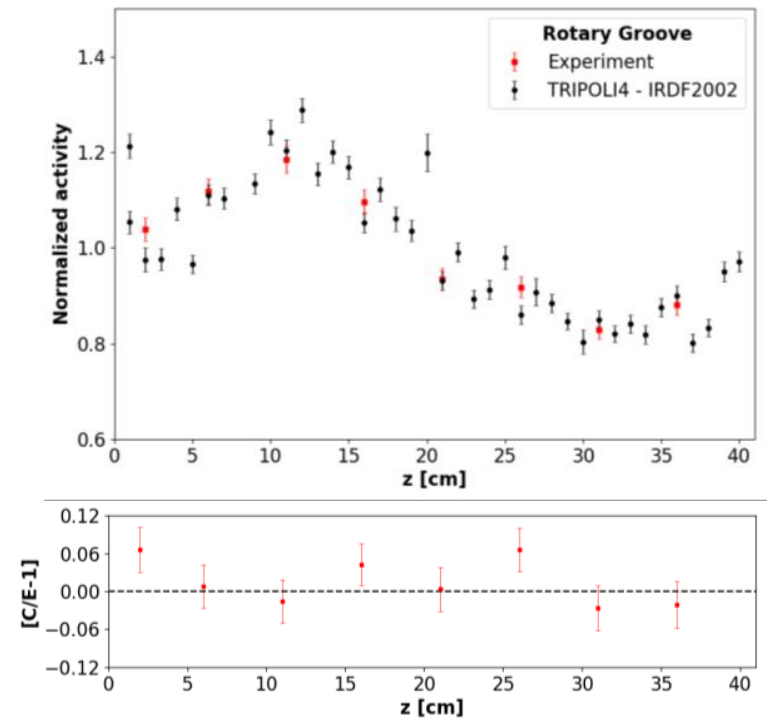

Fig. 11. Measured and calculated ${ }^{197} \mathrm{Au}(\mathrm{n}, \gamma){ }^{198} \mathrm{Au}$ reaction rates in the RG facility normalized to the average value.
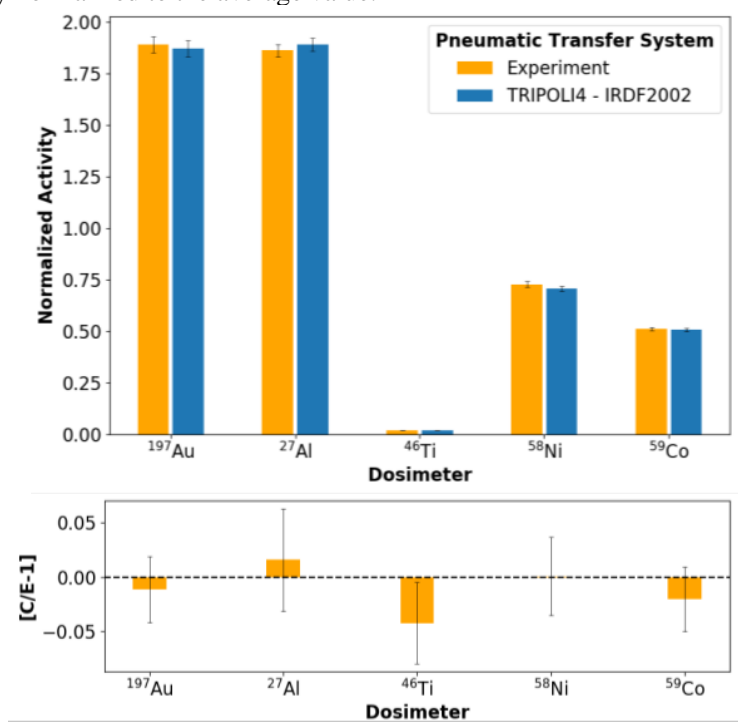

Fig. 12. Measured and calculated reaction rates in the PTS normalized to the ${ }^{197} \mathrm{Au}(\mathrm{n}, \gamma){ }^{198} \mathrm{Au}$ reaction rates in the core center.

IV. CONCLUSION AND PERSPECTIVES

This paper sums up the results of the preliminary analysis of the measurements carried out during the first experimental campaign which was implemented in the TRIGA Mark II reactor of the CNESTEN. Different dosimeters were selected to cover different regions in the energy range of $0-10 \mathrm{MeV}$ of the neutron spectra. The experimental design was accomplished with Monte Carlo simulations using a new detailed computational TRIPOLI-4 model of the reactor TRIGA.
Comparisons, in terms of $\mathrm{C} / \mathrm{E}-1$, have been made between the normalized experimental and calculated reaction rates of the irradiated foils. As a good agreement among the results was deduced, we can assume that the TRIPOLI-4 reactor model is able to correctly reproduce the experimental values.

As a sequel to this work, the spectrum unfolding is going to be achieved by the use of the CALMAR dosimetry adjustment code for neutron spectrum in order to validate the neutron flux and spectra in the irradiation facilities. Moreover, the comparison between absolute values of reaction rates will soon be performed.

\section{REFERENCES}

[1] B. El Bakkari et al., «Monte Carlo modelling of TRIGA research reactor,» Radiation Physics and Chemistry, vol. 79, pp. 1022-1030, 2010.

[2] Y. Boulaich et al., «Steady-state thermal-hydraulic analysis of the Moroccan TRIGA MARK II reactor by using PARET/ANL and COOLOD-N2 codes,» Nuclear Engineering and Design, vol. 241, pp. 270-273, 2011.

[3] E. Chham et al., «Neutronic and thermal-hydraulic analysis of new irradiation channels inside the Moroccan TRIGA Mark II research reactor core,» Applied Radiation and Isotopes, vol. 116, p. 178-184, 2016.

[4] H. El Yaakoubi et at., «Neutronic study of the 2-MW TRIGA MARK-II research reactor by the deterministic codes DRAGON5 \& DONJON5,» Applied Radiation and Isotopes, vol. 157, 2020.

[5] E. Brun, F. Damian, C. Diop, E. Dumonteil, F. Hugot, C. Jouanne, Y. Lee, F. Malvagi, A. Mazzolo, O. Petit, J. Trama, T. Visonneau et A. Zoia, «Tripoli-4®, CEA, EDF and AREVA reference Monte Carlo code,» Annals of Nuclear Energy, vol. 82, pp. 151-160, 2015.

[6] A. Santamarina, D. Bernard, P. Blaise, M. Coste, A. Courcelle, T. Hyunh, C. Jouane, P. Leconte, O. Litaize, S. Mengelle, G. Noguère, J.-M. Ruggiéri, O. Sérot, J. Tommasi, C. Vaglio et J.-F. Vidal, «The JEFF-3.1.1 Nuclear Data Library,» Nuclear Energy Agency Organisation for Economic Co-operation and Development, ISBN 978-92-64-99074-6, OECD , 2009.

[7] N. Soppera, M. Bossant et E. Dupont, «JANIS 4: An improved version of the NEA java-based nuclear data information system,» Nucl. Data Sheets, vol. 120, pp. 294-296, 2014.

[8] N. Soppera, M. Bossant et E. Dupont, «JANIS 4: An Improved Version of the NEA Java-based Nuclear Data information System,» Nuclear Data Sheets, vol. 120, pp. 194-196, 2014.

[9] Zerovnik et al., «On normalization of fluxes and reaction rates in MCNP criticality calculations,» Annals of Nuclear Energy, vol. 63, pp. 126-128, 2014.

[10] «Library for gamma and alpha emissions - CEA/LNHB http://www.lnhb.fr/donnees-nucleaires/module-lara/». 